Systematic assessment with $i$-scan magnification endoscopy and acetic acid improves dysplasia detection in patients with

\title{
Barrett's Esophagus
}

\author{
G Lipman 1,2, R Bisschops 5, V Sehgal 1,2, J0 Fernández-Sordo 3, R Sweis ${ }^{2}$, JM \\ Esteban 4, R Hamoudi 1,6, MR Banks 1,2, K Ragunath 3, LB Lovat 1,2, RJ Haidry \\ 1,2 \\ 1Division of Surgery \& Interventional Science, University College London; ${ }^{2}$ Department of Gastroenterology, University \\ College Hospital NHS Foundation Trust, London; ${ }^{3}$ NIHR Nottingham Digestive Diseases Biomedical Research Unit, \\ Nottingham University Hospitals NHS Trust, ${ }^{4}$ Hospital Clínico San Carlos, Madrid, Spain, ${ }^{5}$ nniversitaire Ziekenhuizen \\ Leuven, Belgium, ${ }^{6}$ Sharjah Institute for Medical Research, College of Medicine, University of Sharjah, Sharjah, UAE.
}

\section{Word Count: 4036 (excluding abstract and references)}

Conflicts of interest: RJH has received research grant support from Pentax Medical, Cook Endoscopy and Covidien plc to support research infrastructure. KR has received research grant support from Pentax Medical

\section{All correspondence to:}

Dr Rehan Haidry, Consultant Gastroenterologist, Director of Endoscopy, 235 Euston Road, London, United Kingdom, NW1 2BU

Email: r.haidry@ucl.ac.uk

Acknowledgements; This work was undertaken at UCLH/UCL who received a proportion of funding from the UK Department of Health's NIHR Biomedical Research Centers funding scheme. The views expressed in this publication are those of the authors and not necessarily those of the Department of Health. The work was also supported by the CRUK UCL Early Cancer Medicine Center.

Abbreviations used in this paper: BE - Barrett's esophagus; EAC - esophageal adenocarcinoma; ACA - acetic acid; HGD - high-grade dysplasia; LGD - low-grade dysplasia; IMC - intramucosal cancer; NBI - Narrow Band Imaging; PPV - positive predictive value; NPV - negative predictive value; PIVI - Preservation and Incorporation of Valuable endoscopic Innovations; ND-BE - non-dysplastic BE; DBE - dysplastic BE; IM - intestinal metaplasia. 


\section{Abstract}

\section{Background and Aims:}

Enhanced endoscopic imaging with chromoendoscopy may improve dysplasia recognition in patients undergoing assessment of Barrett's Esophagus (BE). This may reduce the need for random biopsies detect more dysplasia. The aim of this study was to assess the effect of magnification endoscopy with $i$-Scan (Pentax, Tokyo, Japan) and acetic acid (ACA) on dysplasia detection in BE using a novel mucosal and vascular classification system.

\section{Methods:}

BE segments and suspicious lesions were recorded with high definition (HD) white light and magnification endoscopy enhanced using all $i$-Scan modes in combination. We created a novel mucosal and vascular classification system based on similar previously validated classifications for Narrow band Imaging (NBI). 27 videos were rated before and after ACA application. Following validation, a further 20 patients had their full endoscopies recorded and analysed to model use of the system in a routine clinical scenario to detect dysplasia.

\section{Results:}

The accuracy of the i-scan classification system for BE dysplasia improved with iscan magnification from $69 \%$ to $79 \%$ post-ACA (P= 0.012). Modeling a routine clinical scenario in 20 new patients, accuracy of dysplasia detection rose from $76 \%$ using a 'pull-through' alone to 83\% when ACA and magnification endoscopy were combined $(\mathrm{P}=0.047)$. Overall inter-observer agreement between experts for dysplasia detection was substantial (0.69).

\section{Conclusions:}

We have used a new $i$-scan classification system for $\mathrm{BE}$ and validated against similar systems with NBI with similar outcomes. When our classification is used in combination with magnification and ACA, it can detect BE dysplasia in clinical practice with a good accuracy. 


\section{Introduction}

Esophageal adenocarcinoma (EAC) is the $6^{\text {th }}$ commonest cause of cancer related death in the United Kingdom [1] and the majority of cases are diagnosed at an advanced stage. The only known precursor is Barrett's Esophagus (BE), which carries an estimated annual risk of progression to EAC of $0.3 \%$ [2]. Endoscopic surveillance to detect neoplasia at an early stage [3] permits successful minimally invasive endoscopic intervention [4-6].

The current gold standard for diagnosis remains histology. Guidelines recommend targeted biopsies of suspected areas followed by random sampling every $1-2 \mathrm{~cm}$ throughout the BE segment ('Seattle protocol') [3][7]. Dysplasia can be focal and easily missed since less than $5 \%$ of the BE is actually sampled by the random biopsy technique [8]. Compliance with the Seattle protocol is poor and adherence worsens with longer BE segments [9]. A further drawback is the cost of analysing the large number of biopsies generated with a relatively low yield for dysplasia.

To address these issues, enhanced endoscopic imaging seeks to identify dysplasia optically to allow targeted biopsy only. Enhanced imaging techniques include narrow band imaging (NBI) (Olympus Medical Systems, Tokyo, Japan), FICE (flexible spectral imaging color enhancement) (Fujinon In-telligent Chromo Endoscopy; Fujifilm, Tokyo, Japan), BLI (Blue Laser Imaging) (Lasereo; Fujifilm, Kanagwa, Japan), and $i$-Scan (Pentax, Tokyo, Japan). The most widely available is NBI. Several BE dysplasia classification systems have been suggested using NBI. All focus on 'flat' dysplasia, excluding nodular disease from analysis due to the presumption of dysplasia in these lesions. Between 2006 and 2008, 3 systems were proposed [10-12]. All describe changes in mucosal and vascular patterns following assessment of high quality still images. These basic principles of anomalies arising in vascular and mucosal micro structures in BE have formed the basis of all subsequent pieces of work creating and validating new classification systems with newer enhanced chromoendoscopy technologies. Several studies have validated these systems. Some focused specifically on the quality of the imaging rather than on the accuracy of detection of dysplasia [13-15]. One study 
addressed classification systems using videos [16] but separated sensitivity and specificity for dysplastic and non-dysplastic lesions, so overall parameters are hard to determine. Nevertheless, sensitivity varied from 0.31-0.64 for nondysplastic lesions amongst experts with a specificity of $0.50-0.76$. For dysplastic lesions, these rose to $0.62-0.83$ and $0.64-0.88$ respectively. In a well-designed international randomised controlled trial of NBI compared to routine endoscopy, NBI detected dysplasia with an accuracy of $81 \%$ [17]. This work was similar to work presented by our group in 2014 using $i$-Scan [18].

Recently, a consortium of NBI experts, the BING consortium, developed a simplified consensus driven NBI classification system of BE following a review of 60 NBI magnification images. The mucosa was classified as normal (circular, ridged or tubular pattern) or abnormal (absent or irregular patterns) and the vasculature was either normal (regular vessels with normal or long branching patterns) or abnormal (focally or diffusely distributed vessels following abnormal mucosal architecture). Lesions with abnormal mucosal pattern or vasculature pattern or both were classified as dysplastic. This reported an overall accuracy of 0.85 , sensitivity $0.80 \%$, specificity 0.88 , positive predictive value (PPV) 0.81 and a negative predictive value (NPV) 0.88 , with substantial overall inter-observer agreement $(\kappa=0.68)$. These high quality data have set a benchmark for other technologies to try and match or improve.

Some previous studies have used ACE in combination with virtual chromoendoscopy to generate classifications in BE. Chromoendoscopy with ACA results in an aceto-whitening reaction, highlighting the mucosal pattern and can potentially show the underlying vasculature in areas that have lost acetowhitening. Dysplastic lesions lose aceto-whitening and appear red compared with non-dysplastic areas, permitting targeted assessment and sampling. A recent meta-analysis of 9 studies, calculated a pooled sensitivity of $92 \%$ and specificity of $96 \%$ for the diagnosis of high grade dysplasia or intramucosal cancer [19].

In light of emerging new technologies and the need to reduce unnecessary burden and cost on histopathology services, the Preservation and Incorporation of 
Valuable Endoscopic Innovations (PIVI) initiative of the American Society for Gastrointestinal Endoscopy (ASGE) has set thresholds that any new technology should meet for dysplasia detection in BE before the existing practice of random biopsies can be replaced [20]. These thresholds have been set at a per patient sensitivity $\geq 90 \%$, per patient NPV $\geq 98 \%$ and a specificity $\geq 80 \%$ [20].

A novel virtual chromoendoscopy technology, $i$-Scan (Pentax Hoya, Japan), utilises post-processing technology to provide surface and contrast enhancement in 3 modes (surface enhancement - mode 1, contrast enhancement - mode 2, combined surface and contrast enhancement - mode 3). A new Pentax EG-2990Zi MagniView endoscope permits magnification endoscopy of up to 136 times of the mucosa in all $i$-Scan modes to further characterise the mucosa and micro-vasculature in great detail.

\section{Aims}

The aims of this study were:

- To validate a previously reported Narrow Band Imaging (NBI) classification system using mucosal and vascular structures with i-Scan magnification endoscopy and acetic acid chromoendoscopy.

- To evaluate whether a systematic assessment using ACA and magnification endoscopy improves the accuracy of dysplasia detection with this validated classification system in a routine clinical scenario. 


\section{Methods}

\section{Patients:}

Patients were invited to enrol if they were undergoing surveillance of $\mathrm{BE}$ or endoscopic assessment prior to treatment of dysplasia between January 2013 and January 2015. The study had ethical approval and was registered with ISRCTN (Registration: 58235785). Exclusion criteria included pregnancy, symptomatic esophageal strictures, erosive esophagitis, varices and prior treatment to the esophagus, including endoscopic mucosal resection, radiofrequency ablation or radiotherapy.

Following informed consent, patients underwent routine upper GI endoscopy with a high definition magnification endoscope (Pentax EG-2990Zi MagniView endoscope, $i$-Scan EPK-i7000 High-Definition Video Processor) with a distal cap attachment. This allowed the endoscopists to isolate areas of interest for magnification.

A detailed examination of the BE segment was performed using high definition white light endoscopy (HD-WLE) followed by all $i$-Scan modes. The esophageal mucosa was washed with Simethicone and 2\% N-Acetyl cysteine via a spray catheter. Excess gastric secretions and fluid were aspirated from the stomach. Initial examination was performed using HD-WLE, from the top of the gastric folds to the squamo-columnar junction and the entire esophagus was imaged from the centre of the lumen, this was repeated in $i$-Scan modes 1,2 and 3 (surface enhancement, contrast enhancement and tone enhancement). Each $i$-scan mode delivers unique information about a lesion and therefore when used in combination can provide an overall assessment of a potentially abnormal area. Nodules $\geq 10 \mathrm{~mm}$ or ulcers were excluded, in keeping with similar studies [21]. Areas of suspected dysplasia were then recorded with magnification endoscopy in white light and in all $i$-Scan modes. After all areas of interest had been examined, $2 \%$ ACA was administered and the process repeated. A corresponding biopsy or mucosal resection specimen was taken to confirm pathology from areas of interest and further random biopsies consistent with the Seattle Protocol were taken 
throughout the BE segment. Two expert gastrointestinal histopathologists reviewed all pathological samples.

\section{Utilization of $i$-scan modes and ACA}

i-Scan (Pentax Hoya, Japan), utilises post-processing technology to provide enhancement in 3 modes. The endoscopist can switch between modes in real-time whilst assessing the BE segment with a push of a button on the scope. $i$-Scan 1 , surface enhancement, utilises the technology to detect the edge of a lesion by comparing the difference in pixels and then digitally enhancing the difference resulting in sharper contrast between structures. $i$-Scan 2 , contrast enhancement, detects darker areas of the image and includes blue colour before an algorithm enhances the image resulting in a blueish-white staining of depressed areas and vasculature. $i$-Scan 3 , tone enhancement, dissects and reconstructs the red, green and blue component of each image to provide enhancement of the mucosal structures, vascular patterns and changes in colour. $i$-Scan 1 is often the default setting in clinical practice for lesion detection. $i$-Scan 2 allows for assessment of mucosal and vascular structures in lesion characterization and $i$-Scan 3 enhances glandular structures and is used for lesion demarcation.

In our study, all modes were used in an inter-changeable manner for each video and experts were able to view all lesions allowing them to use the particular enhancement that best suited the lesion in question. Some lesions were only identified with mucosal abnormalities and therefore $i$-Scan 1 was preferred, whereas others had more pronounced vascular abnormalities and therefore $i$-scan 2 or 3 were better. ACA is excellent at highlighting the mucosa though mucosal hyperemia and for identification of abnormal areas with loss of aceto-whitening. However, once an area has lost aceto-whitening, the vasculature remains visible and using $i$-Scan modes 2 and 3 can easily be assessed (as per the examples seen below in Figures 1 and 2) 


\section{Validation of the NBI Classification System Using i-Scan}

Patients undergoing endoscopy at 3 tertiary referral centres (UCLH, Nottingham University Hospital and Universitaire Ziekenhuizen Leuven) were included. Videos were collected targeting individual areas both before and after ACA administration. Videos were converted to MP4 format using Brorsoft Video Converter and edited on an Apple Mac using iMovie software. Clips were exported as $1080 \mathrm{p}$ HD videos. A corresponding biopsy or endoscopic resection specimen was taken from all recorded areas to confirm the histological diagnosis.

Two independent expert GI pathologists reported all biopsies. The consensus report was considered the gold standard. Specimens were graded as NDBE, indefinite for dysplasia (IND), low-grade dysplasia (LGD), or HGD/EAC. Only specimens that confirmed NDBE, HGD or EAC were used to develop the classification system.

A single editor edited all videos. The six experts viewed videos on a HD screen in a randomly assigned order to ensure they were blinded to both diagnosis and pairing of lesions (before and after ACA). Sensitivity, specificity, PPV and NPV was calculated for detection of dysplasia both before and after administration of ACA.

\section{Modeling of Clinical Scenario}

Further videos from 20 patients were collected from the three sites. This time, videos were created from the entire endoscopy rather than from assessments only of individual lesions. Each patient had at least one area identified for targeted magnification endoscopy with a biopsy or mucosal resection. For each patient 4 video clips were viewed in a blinded fashion, a 'pull-through' assessment of the entire BE segment before ACA, a second 'pull-through' after ACA, the lesion before ACA and the lesion after ACA. All experts met in February 2016 and individually viewed the lesion videos on HD iPads at the same time in a random order. After this, the pull-through videos were also displayed in random order. 
Lesions suspicious for dysplasia on 'pull-through' were recorded by experts with a time (seconds into the clip) and location on a clock face (anterior wall lesion = 12 o'clock, right wall = 3 o'clock etc). If no dysplasia was seen, the 'pull-through' was reported as normal.

A clinical scenario was then modeled to represent real time endoscopic assessment. Videos were interpreted as in clinical practice with 4 steps; step 1, a 'pull-through' with $i$-Scan assessment before application of ACA, step 2, focus on any abnormal areas, step 3, a repeat pull-through after application of ACA and step 4, a further assessment of any previously noted areas of interest and any new areas seen after ACA. On a per patient basis the sensitivity, specificity, PPV and NPV was calculated at each of these steps to determine the change in yield when ACA was combined with magnification endoscopy and $i$-Scan enhancement. If no abnormality was detected at step 1 then no detailed assessment of a lesion would occur and the result of step 2 would be ignored. If, however, a lesion was identified at step 1 then all further detailed assessments would be included. Similarly, if a suspicious lesion was noted at any subsequent step, then all further steps would be assessed by the clinician. The process is summarised in figure 3 .

\section{Statistical Analysis}

There are no prior studies of $i$-Scan to detect dysplasia in BE. However, earlier work by our group using $i$-Scan without ACA reported dysplasia was detected with an accuracy of $69 \%$ (Standard deviation 4\%), sensitivity of $67 \%$ (Standard deviation 26\%) and specificity of 69\% (Standard deviation 15\%) [18].

The BING group aimed for a 4\% improvement in the accuracy of diagnosing dysplasia using the NBI classification system with a power of $80 \%$ and assumed a one sided $\alpha$ of 0.05 . Using earlier work from our group, the mean accuracy of the 
$i$-Scan Classification System was 75\% with a SD 0.14. A power calculation was performed to calculate the number of videos required to demonstrate a $4 \%$ improvement in the accuracy of diagnosing dysplasia with a power of $90 \%$. An improvement of $4 \%$ was chosen to mirror the improvement level set by the BING group who also assumed a one-sided significance level of 0.05 [21]. A one-sample $\mathrm{t}$ test power calculation was performed using R/Bioconductor version 3.2 .5 using the power.t.test function [22]. Twenty-seven videos with 6 scorers would be required to determine if the mean accuracy could be improved by $4 \%$, using ACA in the $i$-Scan Classification System.

Sensitivity, specificity, accuracy, PPV and NPV were calculated using the interobserver data. Paired t-test was used to determine significant difference between the groups. Statistical significance cut-off was taken as $p<0.05$. Inter-observer agreement was calculated using k-statistics and a modified Likert scale developed by Landis and Koch [23] was used to interpret $\mathrm{k}$ values (poor $<0.20$; fair $=0.21$ 0.40 , moderate $=0.41-0.60$, substantial $=0.61-0.80$; very good $=0.81-1.00)$. IBM SPSS was used for analyses. Cohen's kappa test and 95\% C.I were calculated using the Kappa.test function in the FSMB library in R/Bioconductor version 3.3.3.

\section{Results}

The expert-group developed a consensus scoring system based on mucosal (M) and vascular (V) patterns: M1 regular oval or villous pits and M2 irregular or featureless mucosa; V1 regular vessels and V2 irregular (dilated or corkscrew) vessels (Table 1; Figures 1 and 2). Predicted dysplasia was calculated based on mucosal and vascular pattern scores (Table 2). This was very similar to all previous classification systems used in BE with NBI [10-12,21].

An area was classified as non-dysplastic only when both normal mucosal and vascular patterns were present (M1V1) (Table 2). 
Validation of NBI Classification System Using i-Scan

For validating our $i$-scan classification against the previous NBI system, 27 lesions were recorded in 21 new patients undergoing assessment for BE dysplasia. Mean age was 69 (range 33-84), 82\% were male. Using Prague criteria, mean circumferential extent of BE was $3 \mathrm{~cm}$ (range $0-12 \mathrm{~cm}$ ) and the mean maximal extent was $5 \mathrm{~cm}$ (range 1-15 cm) (Table 3). Experts rated each video using the classification system defined in phase 1.

Mean video length was 28 seconds for both non-dysplastic BE (ND-BE) and dysplastic BE (D-BE) lesions. The length of videos before and after ACA was not statistically different (29 seconds vs. 27 seconds, $\mathrm{p}=0.25$ ).

Of the 324 video evaluations ( 54 by each of 6 experts), classification was uncertain in only 4.6\%. Reasons included lack of focus and uninterpretable mucosal or vascular pattern. Pathology was predicted as ND-BE or D-BE based on the experts' classification of mucosal and vascular patterns.

ACA improved the experts' mean overall accuracy from $69 \%$ to $79 \%(\mathrm{p}=0.01)$ for dysplasia detection. There was a trend following ACA of improved sensitivity (79\% vs. $87 \%, \mathrm{p}=0.08$ ) and specificity (53\% vs. $68 \%, \mathrm{p}=0.07$ ) of the classification system though neither reached statistical significance. Inter-observer agreement was fair $(\kappa=0.261)$ pre ACA and moderate $(\kappa=0.403)$ after ACA, 95\% C.I 0.03 to 0.83 (Table 4).

Only 2 lesions with LGD were included in the validation phase of the study. All 6 experts scored one lesion abnormal and 5 of the 6 experts scored the second lesion abnormal, the remaining expert felt unable to interpret the clip. 
In the modeling of a clinical scenario, the same experts from the working group reviewed 79 videos (40 clips of pull-throughs and 39 videos of lesions) from 20 patients not previously seen. Experts' diagnosis of ND-BE or D-BE was recorded for each pull-through video and magnification video. Median video length was similar for non-dysplastic and dysplastic pull-throughs (72 seconds vs. 66 seconds, non-paired T-test $\mathrm{p}=0.26$ ) and for non-dysplastic and dysplastic lesions (31 seconds vs. 32 seconds, non-paired T-test $\mathrm{p}=0.65$ ).

No uncertainty was reported in any of the 240 pull-through video evaluations ( 40 by each of 6 experts). Of the 234 lesion assessments (39 by each of 6 experts), $8.1 \%$ were felt to have an uncertain classification. Pathology was again predicted based on classification of mucosal and vascular patterns (Table 2). If a lesion was not classified, the result of the previous step was recorded.

Interpreting the clips as a clinical scenario, the addition of magnification endoscopy (Step 1 versus Step 2) or ACA (Step 1 versus Step 3 or Step 2 versus Step 4) alone did not significantly improve accuracy or sensitivity. However, the complete protocol of magnification endoscopy with the classification system followed by ACA did significantly improve the accuracy. Inter-observer agreement at step 4 of the process was substantial ( $\kappa=0.69$ ), $95 \%$ CI: 0.36 to 1.01 (Table 5).

Sub-group analysis comparing LGD $(n=3)$ and non-dysplastic lesions $(n=8)$ demonstrated similar results. The addition of magnification endoscopy and ACA significantly improved the accuracy and specificity of dysplasia detection and diagnosis (Table 6). 


\section{Discussion}

Early identification of dysplasia through endoscopic surveillance of BE allows patients access to minimally-invasive endoscopic eradication therapy. There is however limited data to show that surveillance in patients with BE impacts significantly on overall survival. The ProBar group examined 783 patients in a multicentre prospective study to examine the clinical benefit of BE surveillance [24]. They concluded EAC was diagnosed at an earlier stage during BE surveillance than in the general population $(\mathrm{p}<0.001)$ and carried a similarly good prognosis to non-surveillance patients diagnosed at the same stage of disease. These findings strengthen the case for routine endoscopic surveillance of BE.

With the on-going advancements in endoscopic imaging it is vital to utilise these tools in a structured manner to give the endoscopist every chance to diagnose dysplasia accurately. $i$-Scan post-processing endoscopic imaging has been shown superior to white light in the detection of IM in BE in previous studies [20]. In our study we used a combination of $i$-Scan enhancements coupled with magnification endoscopy and ACA to allow experts to identify Barrett's associated dysplasia through early changes in mucosal and vascular patterns using a simple classification system that has previously been validated using NBI [21]. Following a systematic assessment of Barrett's Esophagus using ACA and magnification results in an accuracy of $83 \%$, with substantial inter-observer agreement $(\kappa$ $=0.69$ ). These are on par with previous data for similar classification systems.

Several NBI endoscopic classification systems that were developed to identify dysplasia have focused on the mucosal and vascular patterns [10-12]. Evaluation and validation of these systems has relied on the use of selected still images $[14,25]$. Two studies have, however, used videos to validate these classification systems, Baldaque-Silva et al evaluated NBI with optical magnification videos by 6 endoscopists with varying degrees of experience [26]. Dysplasia detection varied from 62-90\% with inter-observer agreement $(\kappa)$ ranging 0.39-0.48. A further study using videos compared classification systems and reported accuracy of 'experts' for diagnosing dysplasia was $0.75-0.78$ with no significant difference 
across the scoring systems. However, global $\kappa$ agreement scores were moderate for the Kansas (0.44) and Amsterdam (0.47) systems and only fair for the Nottingham (0.34) classification system [27].

Recently, a consortium of NBI experts, the BING consortium, developed a simplified consensus driven NBI classification system of BE following a review of 60 NBI magnification images. This was validated with still images and following a web-based survey reported overall accuracy 0.85 , sensitivity $0.80 \%$, specificity 0.88 , positive predictive value (PPV) 0.81 and a negative predictive value (NPV) 0.88 , with substantial overall inter-observer agreement $(\kappa=0.68)$. Of note, analysis was performed on a per-image rather than per-patient basis [21].

Ours is the first clinical study with endoscopic videos to validate this classification system using $i$-Scan technology and demonstrate a benefit in clinical practice through an improvised real time clinical scenario. The scenario was designed to reflect real-life endoscopic assessment of BE; a general view of the BE segment before focusing on areas of suspicion and repeating the process after application of ACA. Magnification endoscopy with $i$-Scan can identify the same mucosal and vascular features described with NBI. Our study validates the use of the NBI classification system with $i$-Scan technology using an international group of expert endoscopists viewing videos collected from several European centres. Addition of ACA significantly raised the accuracy of the classification system using $i$-Scan from $69 \%$ to $79 \%$ ( $\mathrm{p}=0.012)$. Further, in our model of routine clinical practice, accuracy of dysplasia detection was significantly improved with magnification endoscopy and ACA compared to HD-WLE and $i$-Scan alone ( $83 \%$ vs. $76 \%, \mathrm{p}=0.047$ ). Interobserver agreement of the classification system is moderate but when incorporated into a clinical decision making protocol, it improves to substantial ( $\kappa$ $=0.69)$. This later scenario mimics what happens in daily clinical practice and is therefore likely to be a reasonably accurate representation of real life endoscopy. Sub-group analysis of LGD lesions alone generates similar results, with an accuracy of $80 \%$ although these number are very small. 
The results of this study do not meet the current PIVI thresholds for adopting $i$ Scan magnification endoscopy with ACA instead of the current practice of random biopsies. However, this may reflect the low number of lesions reviewed, the prevalence of dysplasia in our sample population and the use of videos rather than still images.

Following an improvised clinical scenario to replicate the real life diagnostic endoscopic approach and analysis on a per-patient basis, $i$-Scan magnification with ACA has a higher sensitivity than the BING study (85\% vs. 80\%) but a lower specificity ( $80 \%$ vs. $88 \%$ ) and accuracy ( $83 \%$ vs. $85 \%$ ), though has similar interobserver agreement (0.69 vs. 0.68) [21]. Direct comparisons are, however, difficult as the BING study and most others of NBI were based on assessment of still images. It is reasonable to assume that the images chosen for analysis in all these studies would have been the best images available. A limitation of the current study like those using still images is the bias introduced by cherry picking optimal views, which may not be achievable in clinical practice. Imaging studies rarely reflect true clinical experience and although this study design has tried to imitate a protocol that can be followed in clinical practice, ultimately, endoscopists can choose to focus on areas of suspicion that may be visible after more than a minute of observation. Nevertheless, this study, based on assessment of videos, is likely to closer represent the real clinical scenario.

It should also be noted that the pull-through videos after ACA were significantly shorter than pre-ACA videos, although this may represent real life clinical practice that less time is spent looking following an initial evaluation.

Previous studies have identified nodules and ulcers in BE as high risk for dysplasia and neoplasia [28]. This and similar studies excluded lesions $>10 \mathrm{~mm}$. However, advances in endoscopic technologies with optical magnification makes previously unrecognised small nodules and ulcers visible, which may bias experts to classify these lesions as dysplastic. The field continues to develop with increasingly good imaging techniques. We suggest that future studies should include assessment of 
small nodules and superficial ulcers to determine whether they improve dysplasia classification.

Future work using the $i$-Scan and NBI classification systems should assess both still images and videos from the same cohort to quantify whether images are a valid reference tool, as most endoscopists rely on video footage rather than stills for diagnostic purposes. A larger study using $i$-Scan technology may enable PIVI thresholds to be met, particularly if micro-nodularity and superficial ulceration are included in the assessment. A further project comparing NBI with $i$-Scan technology may be able to provide a classification system that is validated across all technologies.

In summary, we present data validating the NBI classification system using $i$-Scan endoscopy with magnification endoscopy and ACA. This system has good sensitivity and specificity with fair inter-observer agreement using $i$-Scan. Combining $i$-Scan magnification endoscopy with ACA can be used in a step-wise manner resulting in high accuracy, sensitivity and specificity with substantial inter-observer agreement. The proposed protocol may reduce the need for random biopsies for dysplasia detection. 


\section{$\underline{\text { References }}$}

Office for National Statistics. Available from: http://www.ons.gov.uk/peoplepopulationandcommunity/healthandsocial care/conditionsanddiseases/bulletins/cancerregistrationstatisticsengland /2014

2 Hvid-Jensen F, Pedersen L, Drewes AM, Sørensen HT, Funch-Jensen P. Incidence of adenocarcinoma among patients with Barrett's esophagus. N Engl J Med 2011; 365: 1375-1383 Available from: http://www.ncbi.nlm.nih.gov/pubmed/21995385

3 Fitzgerald $R C$, di Pietro $M$, Ragunath $K$, Ang $Y$, Kang JY, Watson P, Trudgill $N$, Patel $P$, Kaye $P V$, Sanders $S$, O'Donovan M, Bird-Lieberman E, Bhandari $P$, Jankowski J a, Attwood S, Parsons SL, Loft D, Lagergren J, Moayyedi $P$, Lyratzopoulos G, de Caestecker J. British Society of Gastroenterology guidelines on the diagnosis and management of Barrett's oesophagus. Gut 2014; 63: 7-42 Available from: http://www.ncbi.nlm.nih.gov/entrez/query.fcgi?cmd=Retrieve\&db=PubM ed\&dopt=Citation\&list_uids $=24165758$

4 Shaheen NJ, Sharma P, Overholt BF, Wolfsen HC, Sampliner RE, Wang KK, Galanko JA, Bronner MP, Goldblum JR, Bennett AE, Jobe BA, Eisen GM, Fennerty $M B$, Hunter JG, Fleischer DE, Sharma VK, Hawes RH, Hoffman BJ, Rothstein RI, Gordon SR, Mashimo H, Chang KJ, Muthusamy VR, Edmundowicz SA, Spechler $S J$, Siddiqui AA, Souza RF, Infantolino A, Falk GW, Kimmey MB, Madanick RD, Chak A, Lightdale CJ. Radiofrequency ablation in Barrett's esophagus with dysplasia. N Engl J Med 2009; 360: 2277-2288 Available from: http://www.ncbi.nlm.nih.gov/pubmed/19474425

5 Phoa KN, Pouw RE, Bisschops R, Pech O, Ragunath K, Weusten BL a $M$, Schumacher B, Rembacken B, Meining A, Messmann H, Schoon EJ, Gossner L, Mannath J, Seldenrijk C a, Visser M, Lerut T, Seewald S, Ten Kate FJ, Ell C, Neuhaus H, Bergman JJGHM. Multimodality endoscopic eradication for neoplastic Barrett oesophagus: results of an European multicentre study (EURO-II). Gut 2015; 1-8 Available from: http://www.ncbi.nlm.nih.gov/pubmed/25731874

6 Haidry RJ, Butt M a., Dunn JM, Gupta A, Lipman G, Smart HL, Bhandari P, Smith $L$, Willert R, Fullarton G, Di Pietro M, Gordon $C$, Penman I, Barr H, Patel P, Kapoor N, Hoare J, Narayanasamy R, Ang $Y$, Veitch A, Ragunath $K$, Novelli $M$, Lovat LB, Banks M, Sehgal V, Graham D, Sweis R, Miah H, Morris D, Morris J, Boger P, O'Toole D, Cheong E, Direkze N, Dhar A, Nylander D, Mahon B, on behalf of the UK RFA Registry. Improvement over time in outcomes for patients undergoing endoscopic therapy for Barrett's oesophagus-related neoplasia: 6-year experience from the first 500 patients treated in the UK patient registry. Gut 2014; 64: 1192-1199 Available from: http://www.ncbi.nlm.nih.gov/pubmed/25539672\%5Cnhttp://gut.bmj.co $\mathrm{m} /$ cgi/doi/10.1136/gutjnl-2014-

308501\%5Cnhttp://www.pubmedcentral.nih.gov/articlerender.fcgi?artid $=4515987 \&$ tool $=$ pmcentrez\&rendertype $=$ abstract

$7 \quad$ Levine DS, Blount PL, Rudolph RE, Reid BJ. Safety of a systematic endoscopic biopsy protocol in patients with Barrett's esophagus. Am J Gastroenterol 2000; 95: 1152-1157 

$A$, Corley D, Falk G, Goldblum J, Hunter J, Jankowski J, Lundell L, Reid B, Shaheen NJ, Sonnenberg A, Wang K, Weinstein W. A critical review of the diagnosis and management of Barrett's esophagus: The AGA Chicago Workshop. In: Gastroenterology. 2004: 310-330 Abrams JA, Kapel RC, Lindberg GM, Saboorian MH, Genta RM, Neugut AI, Lightdale CJ. Adherence to Biopsy Guidelines for Barrett's Esophagus Surveillance in the Community Setting in the United States. Clin Gastroenterol Hepatol 2009; 7: 736-742

Sharma P, Bansal A, Mathur S, Wani S, Cherian R, McGregor D, Higbee A, Hall $S$, Weston $A$. The utility of a novel narrow band imaging endoscopy system in patients with Barrett's esophagus. Gastrointest Endosc 2006; 64: 167175

Kara MA, Ennahachi M, Fockens P, ten Kate FJW, Bergman JJGHM. Detection and classification of the mucosal and vascular patterns (mucosal morphology) in Barrett's esophagus by using narrow band imaging. Gastrointest Endosc 2006; 64: 155-166

Singh R, Anagnostopoulos GK, Yao K, Karageorgiou H, Fortun PJ, Shonde A, Garsed K, Kaye $P$ V., Hawkey CJ, Ragunath K. Narrow-band imaging with magnification in Barrett's esophagus: Validation of a simplified grading system of mucosal morphology patterns against histology. Endoscopy 2008; 40: 457-463

Alvarez Herrero L, Curvers WL, Bansal A, Wani S, Kara M, Schenk E, Schoon EJ, Lynch CR, Rastogi A, Pondugula K, Weusten B, Sharma P, Bergman JJ, Herrero LA, Curvers WL, Bansal A, Wani S, Kara M, Schenk E, Schoon EJ, Lynch $C R$, Rastogi A, Pondugula K, Weusten B, Sharma P, Bergman JJ. Zooming in on Barrett oesophagus using narrow-band imaging: an international observer agreement study. Eur J Gastroenterol Hepatol 2009; 21: 1068-1075 Curvers WL, Bohmer CJ, Mallant-Hent RC, Naber AH, Ponsioen CIJ, Ragunath $K$, Singh $R$, Wallace MB, Wolfsen HC, Wong Kee Song LM, Lindeboom R, Fockens P, Bergman JJ. Mucosal morphology in Barrett's esophagus: Interobserver agreement and role of narrow band imaging. Endoscopy 2008; 40: 799-805

Curvers W, Baak L, Kiesslich R, Van Oijen A, Rabenstein T, Ragunath K, Rey JF, Scholten P, Seitz U, Ten Kate F, Fockens P, Bergman J. Chromoendoscopy and Narrow-Band Imaging Compared With High-Resolution Magnification Endoscopy in Barrett's Esophagus. Gastroenterology 2008; 134: 670-679 Singh $M$, Bansal a, Curvers WL, Kara $M$ a, Wani SB, Herrero LA, Lynch CR, Kouwen MC a Van, Peters FT, Keighley JD, Rastogi a, Pondugula K, Kim R, Singh V, Gaddam S, Bergman JJ, Sharma P. Observer agreement in the assessment of narrow- band imaging system surface patterns in Barrett ' $s$ esophagus : a multicenter study. Endoscopy 2011; 43: 745-751

Sharma P, Hawes RH, Bansal A, Gupta N, Curvers W, Rastogi A, Singh M, Hall $M$, Mathur SC, Wani SB, Hoffman B, Gaddam S, Fockens P, Bergman JJ. Standard endoscopy with random biopsies versus narrow band imaging targeted biopsies in Barrett's oesophagus: a prospective, international, randomised controlled trial. Gut 2013; 62: 15-21

Sehgal V, Graham D, Banks M, Bisschops R, Ragunath K, Lovat L, Haidry R. A Novel Endoscopic Classification System Using I-Scan Improved Dysplasia 
Setection in Barrett's Oesophagus. 2014: UEG14ABS-4048

Coletta M, Sami SS, Nachiappan A, Fraquelli M, Casazza G, Ragunath K. Acetic acid chromoendoscopy for the diagnosis of early neoplasia and specialized intestinal metaplasia in Barrett's esophagus: a meta-analysis. Gastrointest Endosc 2015;

Sharma P, Savides TJ, Canto MI, Corley DA, Falk GW, Goldblum JR, Wang KK, Wallace MB, Wolfsen HC, ASGE Technology and Standards of Practice Committee. The American Society for Gastrointestinal Endoscopy PIVI (Preservation and Incorporation of Valuable Endoscopic Innovations) on imaging in Barrett's Esophagus. Gastrointest Endosc 2012; 76: 252-254 Available from: http://www.ncbi.nlm.nih.gov/pubmed/22817781 Sharma P, Bergman JJGHM, Goda K, Kato M, Messmann H, Alsop BR, Gupta N, Vennalaganti $P$, Hall $M$, Konda V, Koons A, Penner O, Goldblum JR, Waxman I. Development and Validation of a Classification System to Identify HighGrade Dysplasia and Esophageal Adenocarcinoma in Barrett's Esophagus Using Narrow-Band Imaging. Gastroenterology 2016; 150: 591-598 Ihaka R, Gentleman R. R: A Language for Data Analysis and Graphics. J Comput Graph Stat 1996; 5: 299-314 Available from: http://www.tandfonline.com/doi/abs/10.1080/10618600.1996.1047471 3\#.UvBJfrSOy1N

Landis JR, Koch GG. An application of hierarchical kappa-type statistics in the assessment of majority agreement among multiple observers. Biometrics 1977; 33: 363-374

Kastelein F, van Olphen SH, Steyerberg EW, Spaander MCW, Bruno MJ, ProBar-Study Group. Impact of surveillance for Barrett's oesophagus on tumour stage and survival of patients with neoplastic progression. Gut 2016; 65: 548-554 Available from: http://www.ncbi.nlm.nih.gov/pubmed/25903690 Singh M, Bansal A, Curvers WL, Kara MA, Wani SB, Alvarez Herrero L, Lynch CR, Van Kouwen MCA, Peters FT, Keighley JD, Rastogi A, Pondugula K, Kim R, Singh V, Gaddam $S$, Bergman JJ, Sharma P. Observer agreement in the assessment of narrowband imaging system surface patterns in Barretts esophagus: A multicenter study. Endoscopy 2011; 43: 745-751

Silva FB, Marques $M$, Lunet $N$, Themudo G, Goda K, Toth E, Soares J, Bastos $P$, Ramalho R, Pereira P, Marques N, Coimbra M, Vieth M, Dinis-Ribeiro $M$, Macedo $G$, Lundell L, Marschall $H-U$. Endoscopic assessment and grading of Barrett's esophagus using magnification endoscopy and narrow band imaging: impact of structured learning and experience on the accuracy of the Amsterdam classification system. Scand J Gastroenterol 2013; 48: 160167 Available from: http://www.ncbi.nlm.nih.gov/pubmed/23215965 Silva FB, Dinis-Ribeiro $M$, Vieth $M$, Rabenstein $T$, Goda $K$, Kiesslich $R$, Haringsma J, Edebo A, Toth E, Soares J, Areia M, Lundell L, Marschall HU. Endoscopic assessment and grading of Barrett's esophagus using magnification endoscopy and narrow-band imaging: Accuracy and interobserver agreement of different classification systems (with videos). Gastrointest Endosc 2011; 73: 7-14

Montgomery E, Bronner MP, Greenson JK, Haber MM, Hart J, Lamps LW, Lauwers GY, Lazenby AJ, Lewin DN, Robert ME, Washington K, Goldblum JR. Are ulcers a marker for invasive carcinoma in Barrett's esophagus? Data 
from a diagnostic variability study with clinical follow-up. Am J Gastroenterol 2002; 97: 27-31 Available from: http://www.ncbi.nlm.nih.gov/pubmed/11808966 
Figure 1: SHOWING EXAMPLES OF VARIOUS I-SCAN MODES WITH MAGNIFICATION DEMONSTRAING THE NORMAL (V1) AND ABNORMAL (V2) MICRO-VASCULATURE WITH AND WITHOUT THE ADDITION OF ACETIC ACID.

a) Examples of normal micro-vasculature (V1) as seen with various i-scan modes without acetic acid (regular and uniform microvasculature shown by arrows)

b) Examples of normal micro-vasculature (V1) as seen with various i-scan modes after the addition of acetic acid (regular and uniform microvasculature - shown by arrows). Despite the hyperaemia caused by the addition of ACA one can still make out the underlying regular vessels.

c) Examples of abnormal micro-vasculature (V2) as seen with various i-scan modes without acetic acid (irregular, distorted and tortious microvasculature - shown by arrows)

d) Examples of abnormal micro-vasculature (V1) as seen with various i-scan modes after the addition of acetic acid (irregular, distorted, dilated and tortious microvasculature - shown by arrows). Despite the hyperaemia caused by the addition of ACA one can still make out the underlying irregular vessels.

Figure 2: SHOWING EXAMPLES OF VARIOUS $i$-SCAN MODES WITH MAGNIFICATION DEMONSTRATING THE NORMAL (M1) AND ABNORMAL (M2) MICRO-MUCOSA WITH AND WITHOUT THE ADDITION OF ACETIC ACID.

a) Examples of normal micro-mucosa (M1) as seen with various i-scan modes without acetic acid (regular and uniform mucosal patterns shown by arrows)

b) Examples of abnormal micro-mucosa (M2) as seen with various i-scan modes without acetic acid (irregular, featureless and distorted mucosal patterns - shown by arrows)

c) Examples of normal micro-mucosa (M1) as seen with various i-scan modes with acetic acid (regular and uniform mucosal patterns - shown by arrows)

d) Examples of abnormal micro-mucosa (M2) as seen with various i-scan modes with acetic acid (irregular, distorted and featureless mucosal patterns - shown by arrows)

Figure 3: Modelling of interpretation of dysplasia detection in 4 stages of endoscopic examination to replicate real time clinical decision-making. 
Table 1: $i$-Scan Magnification Classification System for the detection of dysplasia in $\mathrm{BE}$

\begin{tabular}{|l|l|l|}
\hline Mucosal Pattern & M1 & Regular circular or villous pits \\
\hline & M2 & $\begin{array}{l}\text { Distorted or irregular pits OR Featureless } \\
\text { mucosa }\end{array}$ \\
\hline Vascular Pattern & V1 & Regular and uniform vessels \\
\hline & V2 & Irregular, dilated corkscrew vessels \\
\hline
\end{tabular}

Table 2: Interpretation of $\mathrm{M}$ and $\mathrm{V}$ scores

\begin{tabular}{|c|c|}
\hline MV classification & Diagnosis \\
\hline M1 V1 & NO DYSPLASIA \\
\hline M1 V2 & DYSPLASIA \\
\hline M2 V1 & DYSPLASIA \\
\hline M2 V2 & DYSPLASIA \\
\hline
\end{tabular}

Table 3: Patient Characteristics for the Validation of the NBI Classification System Using $i$-Scan

\begin{tabular}{|l|l|}
\hline Number of Patients & 21 \\
\hline Number of lesions recorded & 27 \\
\hline Mean Age (range) & $69(46-83)$ \\
\hline Male & $86 \%$ \\
\hline Mean Barrett's Circumferential Length (cm, & $3(0-12)$ \\
\hline range) & \\
\hline Mean Barrett's Maximal Length (cm, range) & $5(1-15)$ \\
\hline
\end{tabular}


Table 4: Validation of the $i$-Scan Classification System for BE

\begin{tabular}{|c|c|c|c|}
\hline & Mean Pre ACA & Mean Post ACA & Paired t-test p value \\
\hline Accuracy & $69 \%$ (SD 13\%) & $79 \%$ (SD 11\%) & 0.012 \\
\hline Sensitivity & 79\% (SD 10\%) & $87 \%(S D 14 \%)$ & 0.081 \\
\hline Specificity & $53 \%($ SD $23 \%$ ) & $68 \%$ (SD 21\%) & 0.067 \\
\hline PPV & 73\% (SD 11\%) & $81 \%$ (SD 13\%) & 0.112 \\
\hline NPV & $63 \%$ (SD 16\%) & $81 \%$ (SD 17\%) & 0.016 \\
\hline Cohen's к & 0.261 & 0.403 & \\
\hline $95 \%$ C.I & -0.18 to 0.68 & 0.03 to 0.83 & \\
\hline
\end{tabular}


Table 5: Results for each step of the protocol as per the clinical modelling scenario (Step 1, a pull-through, Step 2, a focus on any abnormal area, Step 3, a pull-through after application of ACA and Step 4, a further assessment of any previously noted areas of interest and any new areas seen after ACA) in all patients.

\begin{tabular}{|c|c|c|c|c|c|}
\hline & Step 1 & Step 2 & Step 3 & Step 4 & $\begin{array}{c}\text { Paired t-test } \\
\text { P value } \\
\text { Step } 1 \text { vs. Step } 4\end{array}$ \\
\hline Accuracy & $76 \%$ & $77 \%$ & $75 \%$ & $83 \%$ & 0.047 \\
\hline Sensitivity & $91 \%$ & $81 \%$ & $94 \%$ & $85 \%$ & 0.19 \\
\hline Specificity & $57 \%$ & $74 \%$ & $52 \%$ & $80 \%$ & 0.0008 \\
\hline PPV & $72 \%$ & $77 \%$ & $70 \%$ & $84 \%$ & 0.005 \\
\hline NPV & $84 \%$ & $78 \%$ & $88 \%$ & $81 \%$ & 0.63 \\
\hline Cohen's к & 0.667 & 0.365 & 0.679 & 0.690 & \\
\hline 95\% C.I. & $0.22-1.09$ & $0.18-1.0$ & 0.28-1.09 & $0.36-1.01$ & \\
\hline
\end{tabular}

Table 6: Results for each step of the protocol as per the clinical modelling scenario (Step 1, a pull-through, Step 2, a focus on any abnormal area, Step 3, a pull-through after application of ACA and Step 4, a further assessment of any previously noted areas of interest and any new areas seen after ACA) in all patients with LGD alone.

\begin{tabular}{|c|c|c|c|c|c|}
\hline & Step 1 & Step 2 & Step 3 & Step 4 & $\begin{array}{l}\text { Paired t-test } P \\
\text { value } \\
\text { Step } 1 \text { vs. Step } 4\end{array}$ \\
\hline Accuracy & $64 \%$ & $68 \%$ & $63 \%$ & $80 \%$ & $<0.001$ \\
\hline Sensitivity & $78 \%$ & $57 \%$ & $94 \%$ & $88 \%$ & 0.17 \\
\hline Specificity & $59 \%$ & $71 \%$ & $52 \%$ & $78 \%$ & 0.003 \\
\hline PPV & $39 \%$ & $36 \%$ & $40 \%$ & $56 \%$ & 0.04 \\
\hline NPV & $89 \%$ & $85 \%$ & $97 \%$ & $95 \%$ & 0.12 \\
\hline Cohen's к & 0.657 & 0.25 & 0.625 & 0.621 & \\
\hline $95 \%$ CI & $0.22-1.09$ & $-0.35-0.85$ & $0.15-1.09$ & $0.22-1.09$ & \\
\hline
\end{tabular}

